$\xi$

\title{
Effects of Slip, Brownian Motion and Thermophoresis on Peristaltic Pumping of Nano Fluid in an Asymmetric Channel with Porous Medium
}

\author{
S.Sreenadh ${ }^{1}$, G.Yasodhara ${ }^{2}$, B.Sumalatha ${ }^{3}$ and A.N.S.Srinivas ${ }^{4 *}$ \\ ${ }^{1,3}$ Department of Mathematics, Sri Venkateswara University, Tirupati, 517 502, A.P., India. \\ ${ }^{2}$ Department of Mathematics, Government Junior College for Girls, Chandragiri, Chittor, A.P., India \\ ${ }^{4}$ Department of Mathematics, School of Advanced Sciences, VIT, Vellore, 632 014, T.N., India. \\ *Corresponding authorE-mail: anssrinivas@vit.ac.in, akkirajusrinivas@gmail.com
}

\begin{abstract}
This paper deals with peristaltic motion of electrically conducting nanofluid in a tapered asymmetric channel through a porous medium in presence of heat and mass transfer under the effect of slip conditions. The problem is reduced mathematically by a set of nonlinear partial differential equations which describe the conservation of mass, momentum, energy and concentration of nanoparticles. The nondimensional form of these equations is simplified under the assumption of long wavelength and low Reynolds number. The coupled governing equations are solved analytically. The expressions for velocity, stream function, temperature and concentration are derived. The results have been presented graphically for the various interested emerging parameters and the obtained results are discussed in detail. It is observed that the magnitude of the velocity decreases in the middle of the channel while it increases near the channel walls with an increase in the non-uniform parameter $m$. It is also noticed that the nanoparticle temperature increases with increasing thermal slip parameter $L_{2}$. The present result coincides with the findings of Kothandapani and Prakash [19].
\end{abstract}

Keywords: Peristaltic motion, Nanoparticles, tapered asymmetric channel, MHD.

\section{Introduction}

In recent years, peristaltic pumping plays an important role in development of Science and Engineering. A variety of complex rheological fluids can easily be transported from one place to another place with a special type of pumping known as peristaltic pumping. Peristaltic flows are generated by the propagation of waves along the flexible walls of the channel or tube. This mechanism is seen in many biological system such as urine transport from kidney to bladder through the ureter, transport of lymph in the lymphatic vessels, swallowing food through the esophagus, the movement of chime in the gastrointestinal track, ovum movement in the fallopian tube, transport of spermatozoa vasomotion of small blood vessels etc. This analysis was first investigated by Latham [1]. Later, Jaffrin and Shapiro [2] investigated the peristaltic pumping under the assumption of long wavelength and low Reynolds number approximation. This work was further extended by so many researchers. Several theoretical and experimental studies have been reported by many of the researchers on peristaltic motion of different physiological fluids with different flow geometries and boundary conditions [3-12].

Nowadays, the study of nanofluids flow has the interest of researchers because of its large number applications in biomedical and industrial engineering. Choi and Eastman [13] was the first who initiated this nanofluid technology. The fundamental features are thermal conductivity enhancement. A detailed analysis of nanofluids was discussed by Buongiorno [14]. According to his view the massive increase in thermal conductivity occurs due to the presence of the Brownian diffusion and the thermophoresis diffusion of the nanoparticles. Sheikholeslami et al. [15] studied the natural convection in a concentric annulus between a cold outer square and heated inner circular cylinders in the presence of static radial magnetic field. Noreen [16] investigated the mixed convection peristaltic flow of third order nanofluid with an induced magnetic field and observed that the influence of Brownian motion parameter and thermophoresis parameter on mass distribution is opposite to temperature distribution. Ebaid and Emand [17] gave an exact analytical solution of the peristaltic nanofluid flow in an asymmetric channel with flexible walls and slip condition which is useful for Application to the cancer treatment. Nadeem et al. [18] analyzed the endoscopic effects of peristaltic nanofluid flow of Jeffrey six-constant fluid model in the presence of magneto hydrodynamics flow. Kothandapani and Prakash [19] studied on peristaltic transport of carreau nanofluid under effect of a magnetic field in a tapered asymmetric channel. Dhanapal et al. [20] examined the peristaltic flow of an incompressible micropolar nanofluid in a tapered asymmetric channel in the presence of thermal radiation, heat source parameters and noticed that the coupling number and Brownian motion parameters have opposite effects on the microrotation velocity. Prakash and Suriyakumar [21] analyzed the effect of uniform transverse magnetic field on the transient free convective nanofluid flow of an incompressible, viscous electrically conducting fluid between vertical asymmetric parallel plates in the presence of internal heat generation. They observed that the increase in Grashof number of copper-water and alumina-water nanofluids lead to increase in the velocity of the flow field. Eldabe et al. [22] studied the peristaltic motion of a 
Carreau nano fluid model through a porous medium inside an asymmetric channel. It is found that the velocity of Carraeu nanofluid increases at the neighborhood of the channel walls and decreases near the center of the channel by increasing the Weissenberg number and the power law index. Recently, Mishra et al. [23] investigated the heat and mass transfer in asymmetric channels during peristaltic transport of an MHD fluid having temperature-dependent properties by using perturbation technique. It is found that when the fluid viscosity diminishes, the size of the bolus increases.

According to the observation of many physiologists, intra-uterine fluid flow due to myometrial contractions, we made an attempt to study the peristaltic transport of electrically conducting nanofluid in a tapered asymmetric channel through a porous medium in presence of heat and mass transfer under the effect of slip conditions. A detailed mathematical formulation is presented. Expressions for the stream function, velocity, temperature and concentration have been derived and discussed through graphs. As thermal, velocity and concentration slip parameters and permeability parameter tends to zero our results agree with the results of $\mathrm{Ko}$ thandapani and Prakash [19].

\section{Mathematical Formulation of the Problem}

Consider the peristaltic flow of an incompressible viscous electrically conducting nanofluid in a two dimensional infinite asymmetric channel through porous medium in presence of heat and mass transfer under the effect of slip conditions. The flow is generated by sinusoidal wave trains propagating with constant speed $c$ along the walls to have different amplitudes and phases. The shapes of the channel walls are presented as:

$$
\begin{aligned}
& H_{1}=-a_{1} \sin \left[\frac{2 \pi}{\lambda}(\bar{X}-c \bar{t})+\phi_{0}\right]-\bar{m} \bar{x}-d \\
& H_{2}=a_{2} \sin \left[\frac{2 \pi}{\lambda}(\bar{X}-c \bar{t})\right]+\bar{m} \bar{x}+d
\end{aligned}
$$

$\lambda$ is the wavelength, $t$ is he time and $c$ is the propagation velocity.

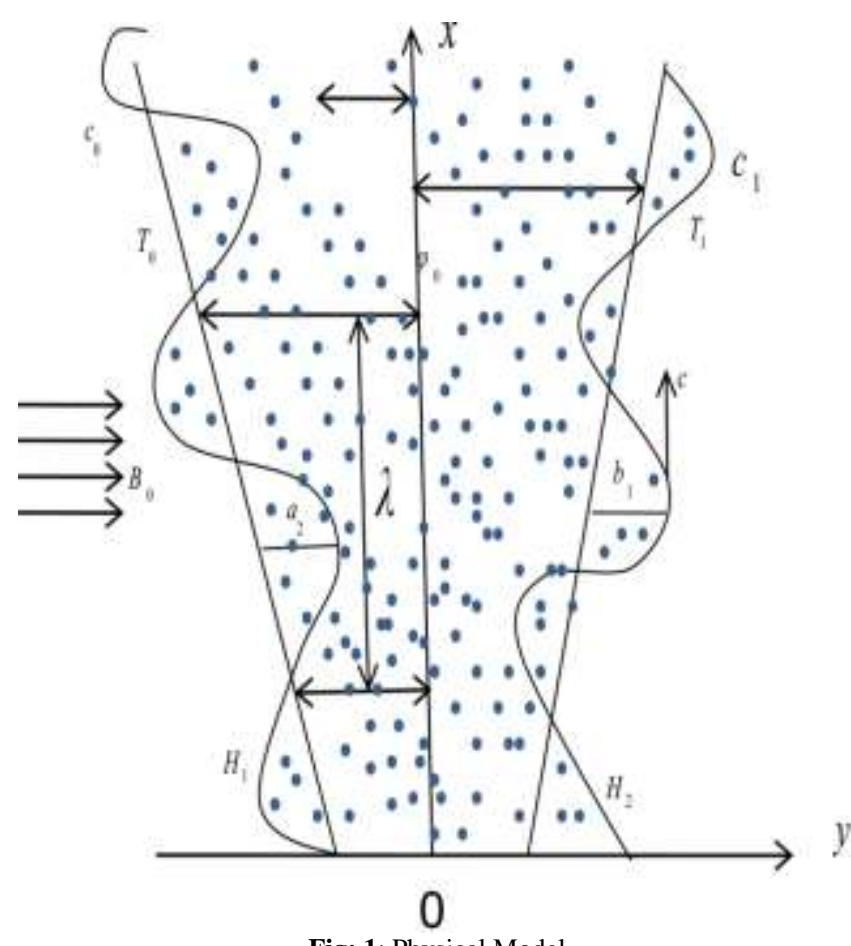

Fig: 1: Physical Model
Here the amplitudes of the waves, width of the channel and phase difference $a_{1}, a_{2}, 2 d, \phi_{0}$ satisfy the condition for the divergent channel at the inlet of flow

$a_{1}^{2}+a_{2}^{2}+2 a_{1} a_{2} \cos (\phi) \leq 4 d^{2}$

A uniform magnetic field with magnetic flux density $\mathbf{B}=\left(0,0, B_{0}\right)$ is applied. The heat transfer and nanoparticle processes are maintained by considering temperature $T_{0}, T_{1}$ and nanoparticle phenomenon $C_{0}, C_{1}$ to the right and left side walls respectively.

The non-dimensional quantities are given below:

$h_{1} d=H_{1}, h_{2} d=H_{2}, x \lambda=\bar{x}, a d=a_{1}, b d=a_{2}, m d=\lambda \bar{m}, \psi c d=\bar{\psi}$,

$u c=\bar{u}, t \lambda=c \bar{t}, \delta \lambda=d, N t=\frac{\tau D_{T}\left(T_{1}-T_{0}\right)}{T_{m} v}, N b=\frac{\tau D_{B}\left(C_{1}-C_{0}\right)}{v}$,

$p=\frac{d^{2} \bar{p}}{\mu c \lambda}, M=\sqrt{\frac{\sigma}{\mu}} B_{0}, \operatorname{Re}=\frac{\rho_{f} c d}{\mu}, \theta=\frac{\left(T-T_{0}\right)}{\left(T_{1}-T_{0}\right)}, \phi=\frac{\left(C-C_{0}\right)}{\left(C_{1}-C_{0}\right)}$,

$G r_{t}=\frac{\left(1-C_{0}\right) \rho_{f} g \alpha d^{2}\left(T_{1}-T_{0}\right)}{c \mu}, G r_{c}=\frac{\left(\rho_{p}-\rho_{f}\right) \rho_{f} g \beta d^{2}\left(C_{1}-C_{0}\right)}{c \mu}$

$\operatorname{Pr}=\frac{\rho \mu c_{f}}{k_{0}}, R n=\frac{16 \sigma^{*} T_{0}^{3}}{3 K \mu c_{f}}, \sigma=\frac{d}{\sqrt{k}}$

Kothandapani and Prakash [19] gave the following simplified nondimensional governing equations that governs the fluid flow under the assumption of low Reynolds number and long wave length approximation:

$$
\begin{aligned}
& \frac{\partial p}{\partial x}=\frac{\partial^{3} \psi}{\partial y^{3}}-\left(M^{2}+\sigma^{2}\right) \frac{\partial \psi}{\partial y}+G_{r} \theta+G_{c} \phi \\
& \frac{\partial p}{\partial y}=0
\end{aligned}
$$

$\frac{\partial^{2} \theta}{\partial y^{2}}+\frac{\operatorname{Pr} N b}{1+\operatorname{Pr} R n}\left(\frac{\partial \theta}{\partial y}\right)\left(\frac{\partial \phi}{\partial y}\right)+\frac{\operatorname{Pr} N t}{1+\operatorname{Pr} R n}\left(\frac{\partial \theta}{\partial y}\right)^{2}$

$\frac{\partial^{2} \phi}{\partial y^{2}}+\frac{N t}{N b} \frac{\partial^{2} \theta}{\partial y^{2}}=0$

The corresponding boundary conditions are

$\psi=\frac{F}{2}, \frac{\partial \psi}{\partial y}+L_{1} \frac{\partial^{2} \psi}{\partial y^{2}}=-1$,

$\theta+L_{2} \frac{\partial \theta}{\partial y}=1, \phi+L_{3} \frac{\partial \phi}{\partial y}=1$ at $y=h_{2}=b \sin [2 \pi(x-t)]+1+m x$

$\psi=-\frac{F}{2}, \frac{\partial \psi}{\partial y}-L_{1} \frac{\partial^{2} \psi}{\partial y^{2}}=-1$,

$\theta-L_{2} \frac{\partial \theta}{\partial y}=0, \phi-L_{3} \frac{\partial \phi}{\partial y}=0$ at $y=h_{1}=-a \sin \left[2 \pi(x-t)+\phi_{0}\right]-1-m x$

The relation between non-dimensional mean flows $F$ and $\Theta$ is given by

$$
F=\Theta+\sin [2 \pi(x-t)] a+\sin [2 \pi(x-t)+\phi] b
$$

where $F=\int_{h_{1}}^{h_{2}} u d y=\int_{h_{1}}^{h_{2}} \frac{\partial \psi}{\partial y} d y$ 


\section{Solution of the Problem:}

With the help of boundary conditions in equations (9), the solutions of the equations (5)-(8) are

$$
\begin{aligned}
& \theta=p_{2}+p_{3} e^{-m_{1} p_{1} y} \\
& \phi=-\frac{N t}{N b} p_{3} e^{-m_{1} p_{1} y}+p_{1} y+p_{4} \\
& \psi=p_{5}+p_{6} y+p_{7} \cosh T y+p_{8} \sinh T y+m_{2} e^{-m_{1} p_{1} y}+m_{3} y^{2} \\
& u=p_{6}+T p_{7} \sinh T y+T p_{8} \cosh T y-m_{1} m_{2} p_{1} e^{-m_{1} p_{1} y}+2 m_{3} y
\end{aligned}
$$

Values of the constants are given in the appendix - A.

\section{Results and Discussions:}

In order to get insight into the given physical problem, the behaviors of various parameters are discussed on temperature, velocity and concentration. The effects of significant physical parameters on the flow phenomenon are discussed through graphs by using the fixed values of the parameter $a=0.12, b=0.2, M=1, N t=2, N b=3, R n=1$,

$\operatorname{Pr}=1, \sigma=1, G r=1.5, G c=0.5, x=0.6, t=0.1, m=0.2$,

$L_{1}=0.1, L_{2}=0.1, L_{3}=0.1, \Theta=1.5, \Phi_{0}=\frac{\pi}{4}$. In this section we have presented the numerical results to analyze the impact of nonuniform parameter $(m)$, thermophoresis parameter $(N t)$, Brownian motion parameter $(N b)$, Prandtl number $(\mathrm{Pr})$, Reynolds number $(R n)$, thermal slip parameter $\left(L_{2}\right)$, concentration slip parameter $\left(L_{3}\right)$, temperature Grashof number $(G r)$, velocity slip $\left(L_{1}\right)$, magnetic parameter $(M)$, mass Grashof number $(G c)$,wave frame mean flow $(\Theta)$, permeability parameter $(\sigma)$ on temperature, concentration and velocity profiles through graphs and are shown from figures (2) $-(21)$.

\subsection{Temperature Profiles:}

From Figure 2, it is noticed that when there is an increase in the values of non - uniform parameter $m$ increases the temperature profiles in left wall of the channel. Figures 3-7, it is clear that the temperature profiles increases when there is an increase in $N t, N b, \operatorname{Pr}$ and $L_{2}$, however it decreases with the increase in $R n$.

\subsection{Concentration Profiles:}

From figure 8 , it is found that the concentration profiles increases in the left half of the channel and decreases in the right half of the channel with the increasing values of non-uniform parameter $m$. Figures 9-13 depicts that increase in $N t, \operatorname{Pr}$ and $L_{3}$ reduces the concentration profiles whereas reverse trend is observed in the case of $N b$ and $R n$.

\subsection{Velocity Profiles:}

Figures 14-17, depicts the velocity region reduction in the left half of the channel and increases in the right half of the channel with increasing magnitude of $G r, L_{1}, G c$ and $N t$. The influence of $M$ and $\sigma$ on the velocity is presented in the Figures 18 and 19 It is found that the increasing values of $M$ and $\sigma$ reduces the velocity in the middle of the channel and enhances the velocity at the walls of the channel. From figure 20, it is observed that the velocity enhances in the left of the channel and diminishes in the right half of the channel with the increasing magnitude of Brownian motion parameter. Figure 21 shows that the increase in flow rate $\Theta$ improves the velocity.

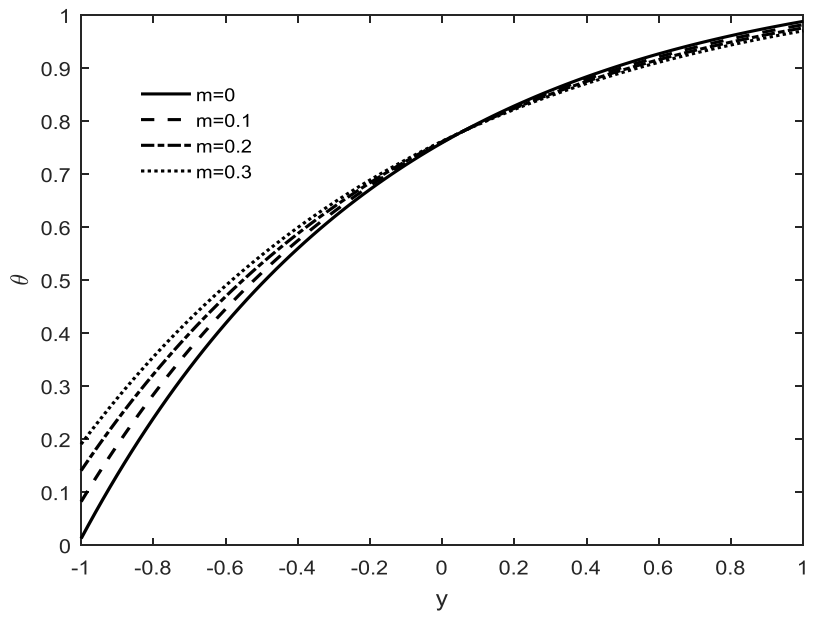

Fig. 2: Influence of non-uniform parameter on temperature profiles

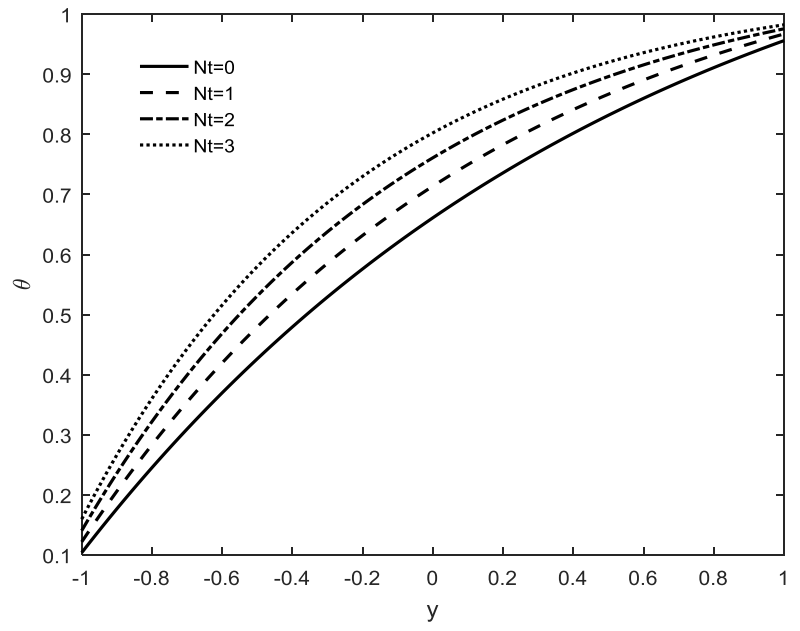

Fig. 3: Influence of Thermophoresis parameter on temperature profiles.

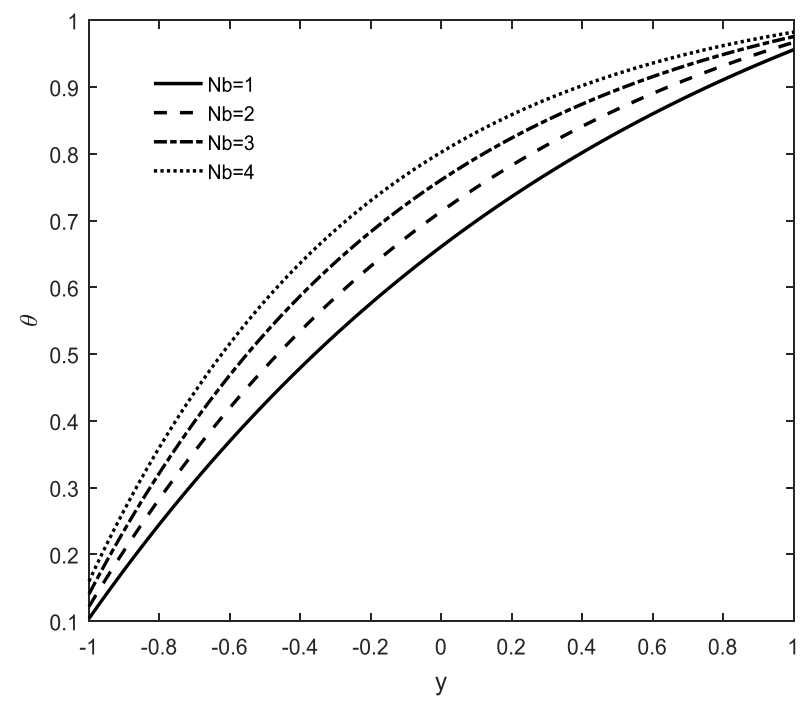

Fig. 4: Influence of Brownian motion parameter on temperature profiles 


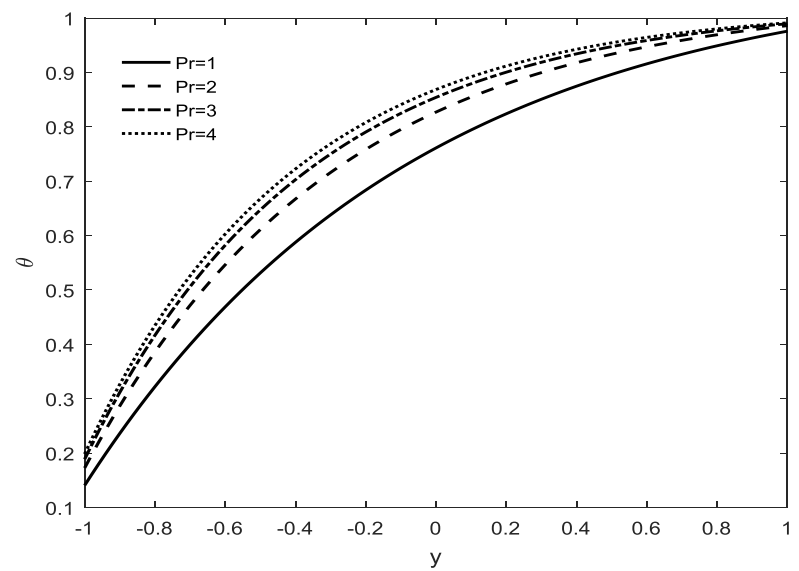

Fig. 5: Influence of Prandtl number on temperature profiles

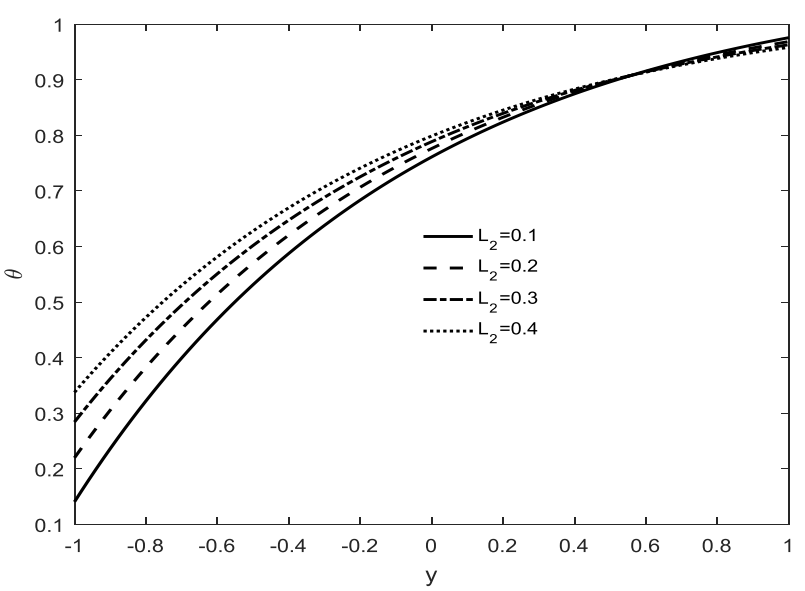

Fig. 6: Influence of thermal slip parameter on temperature profiles

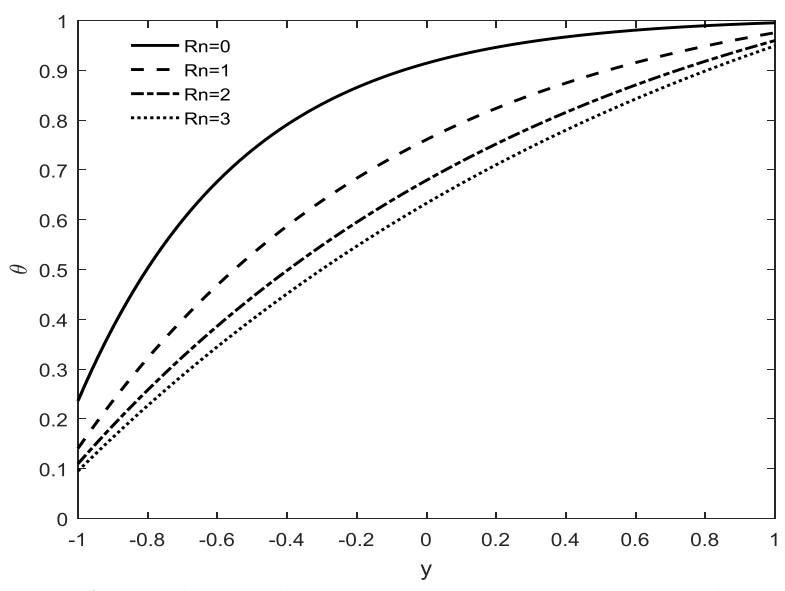

Fig. 7: Influence of Reynolds number on temperature profiles.

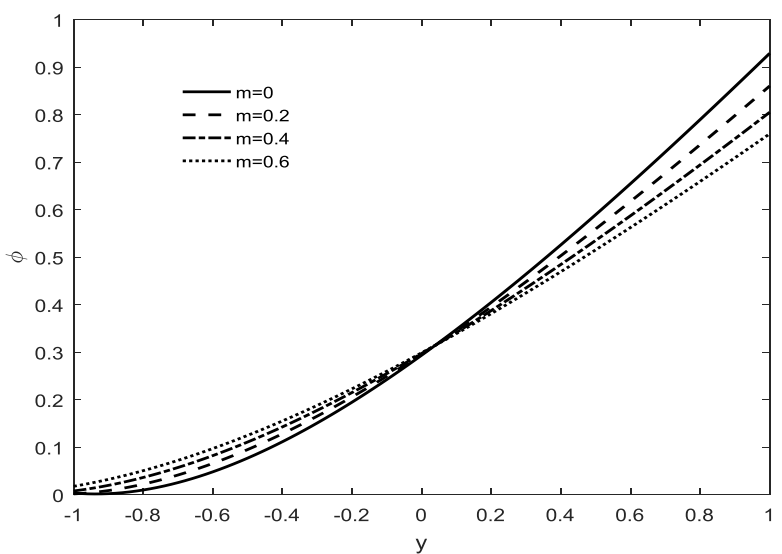

Fig. 8: Influence of non-uniform parameter on concentration profiles.

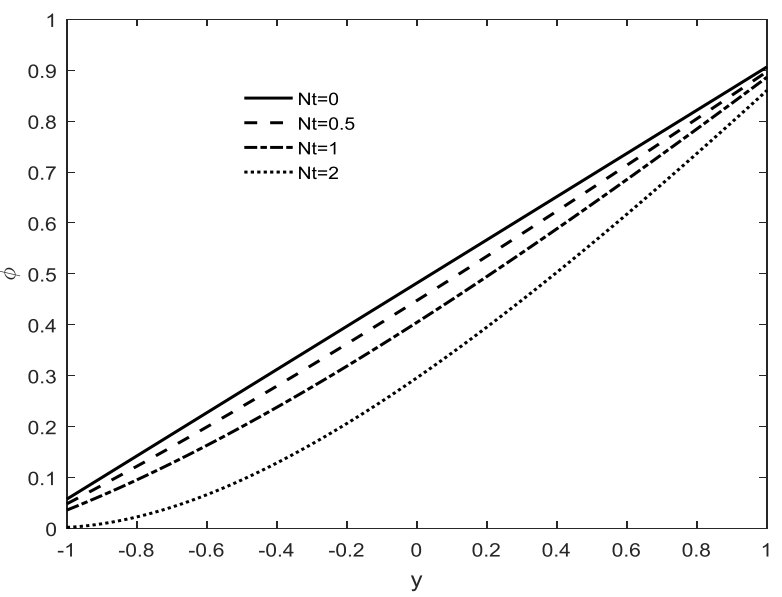

Fig. 9: Influence of Thermophoresis parameter on concentration profiles

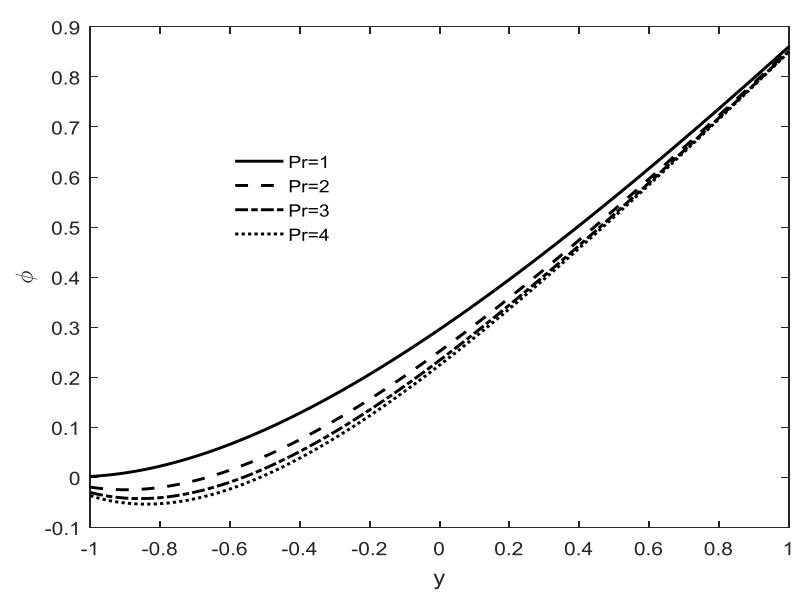

Fig. 10: Influence of Prandtl number on concentration profiles

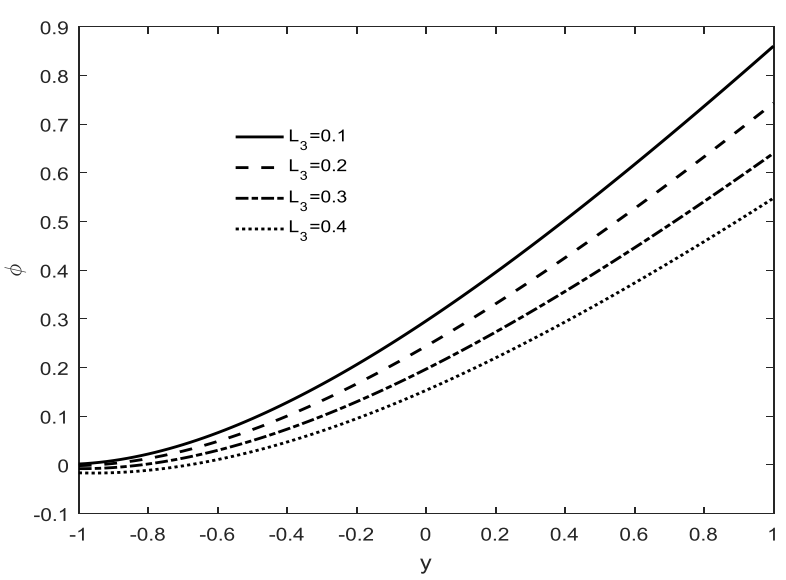

Fig. 11: Influence of concentration parameter on concentration profiles

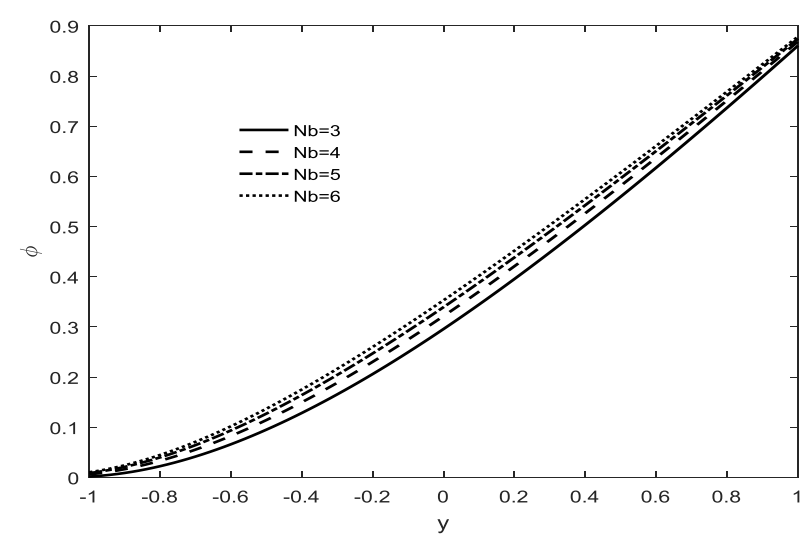

Fig.12: Influence of Brownian motion parameter on concentration profiles 


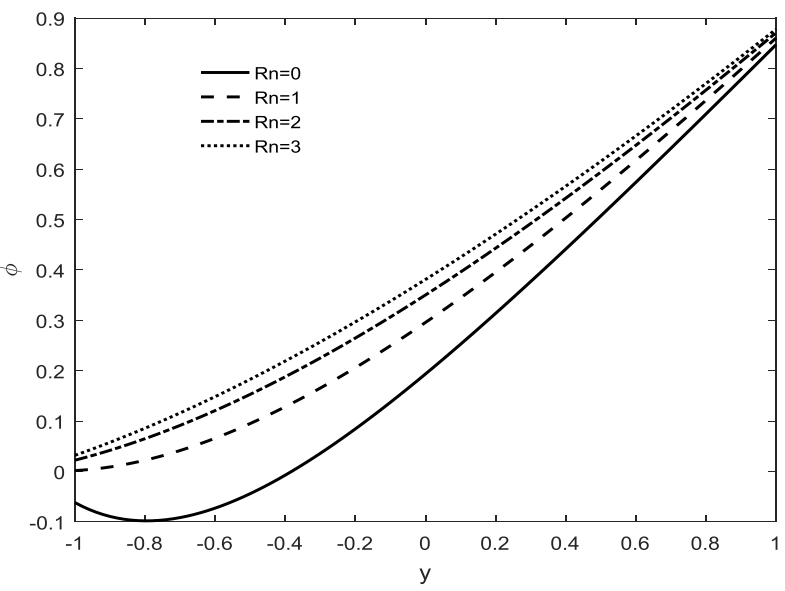

Fig. 13: Influence of Reynolds number on concentration profiles

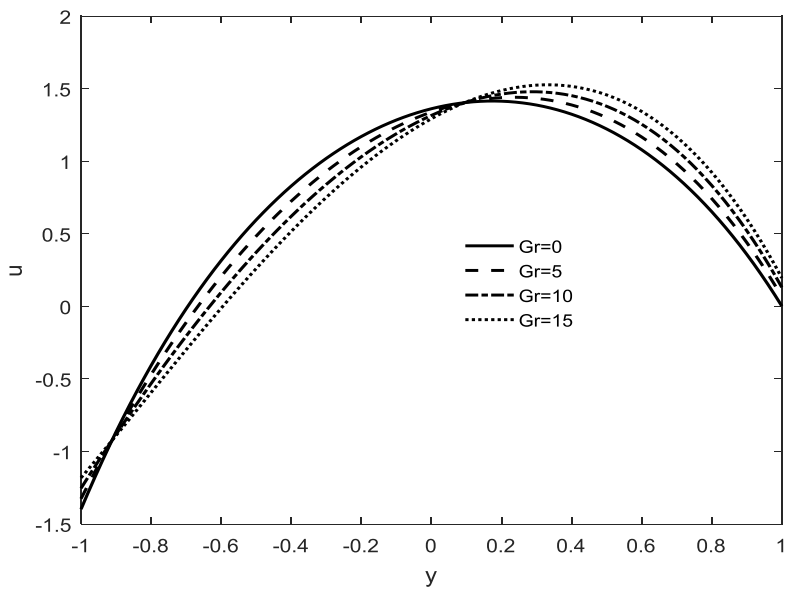

Fig.14: Influence of thermal Grashof number on velocity profiles.

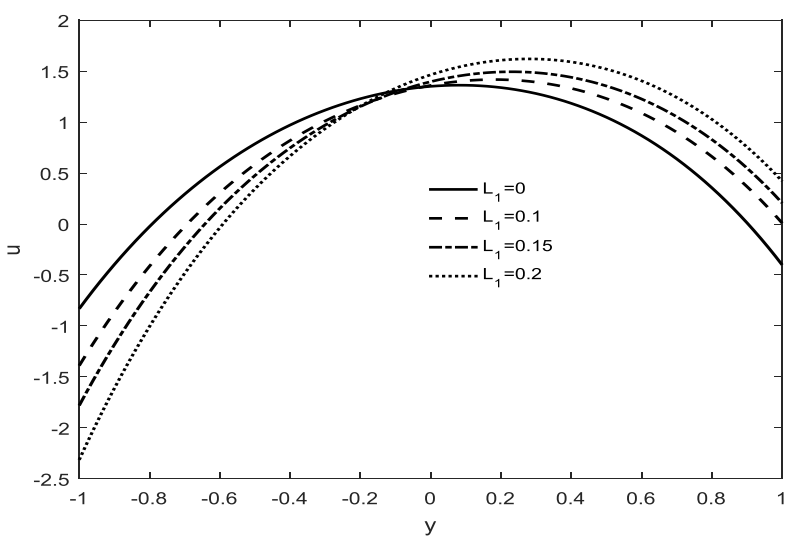

Fig. 15: Influence of velocity slip parameter on velocity profiles.

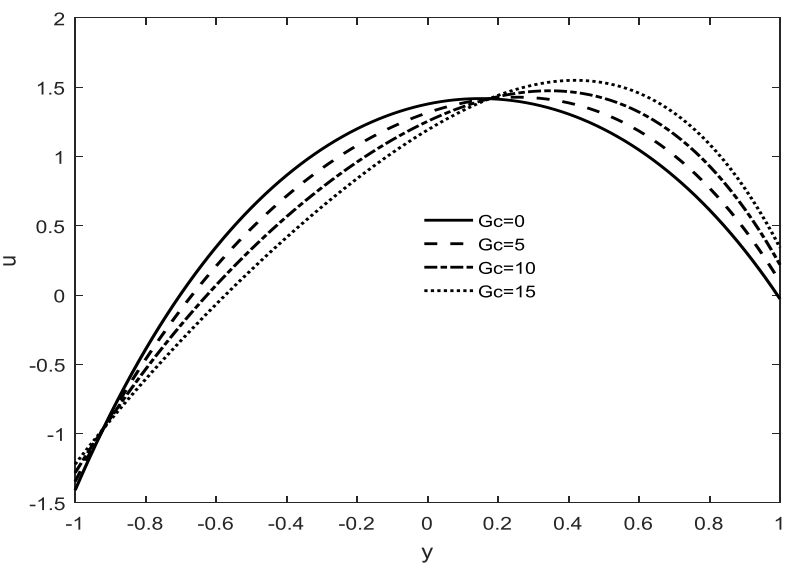

Fig. 16: Influence of mass Grashof number on velocity profiles

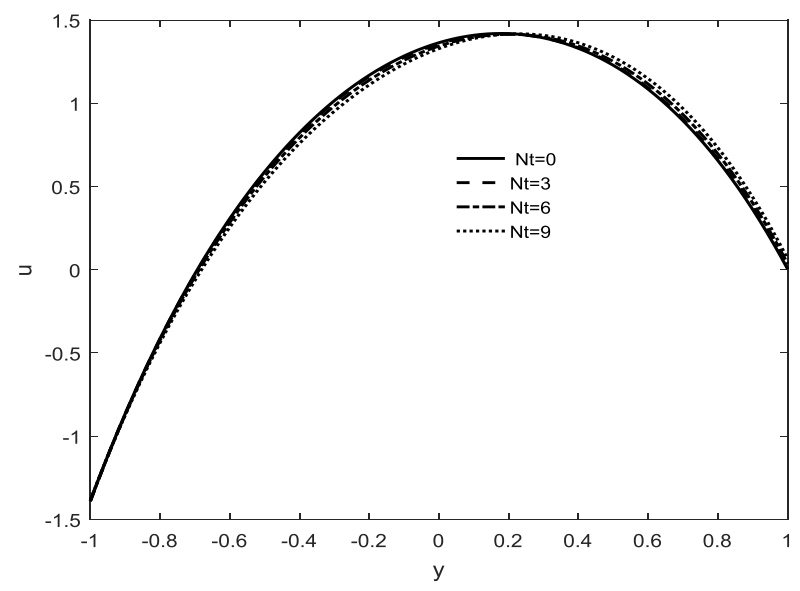

Fig. 17: Influence of thermophoresis parameter on velocity profiles

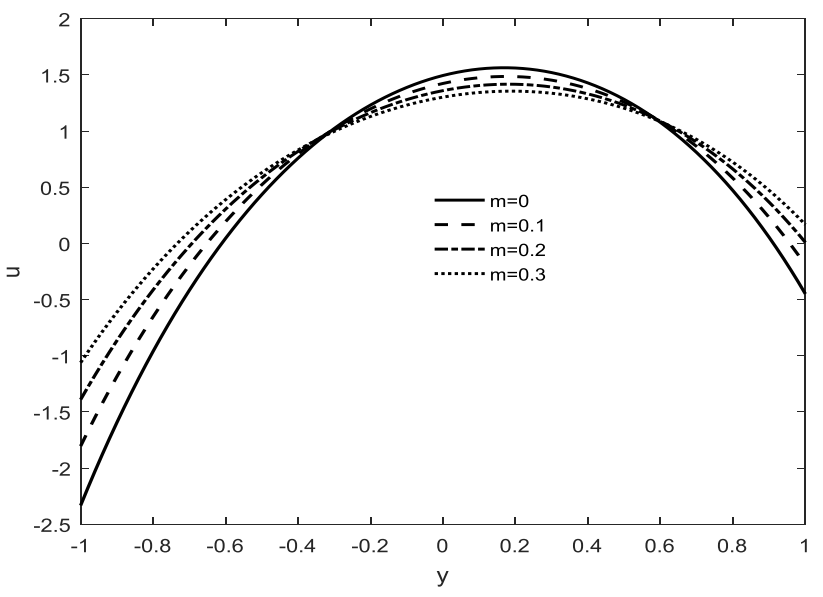

Fig. 18: Influence of non-uniform parameter on velocity profiles

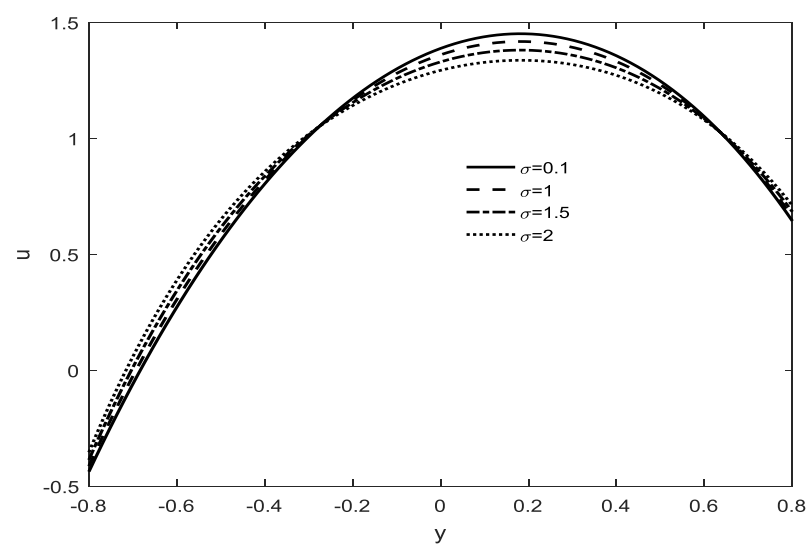

Fig. 19: Influence of permeability parameter on velocity profiles.

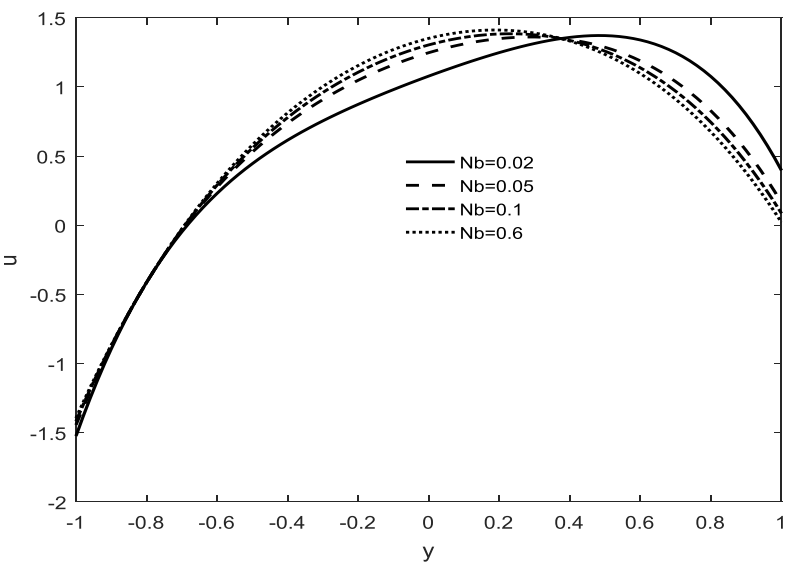

Fig. 20: Influence of Brownian motion parameter on velocity profiles. 


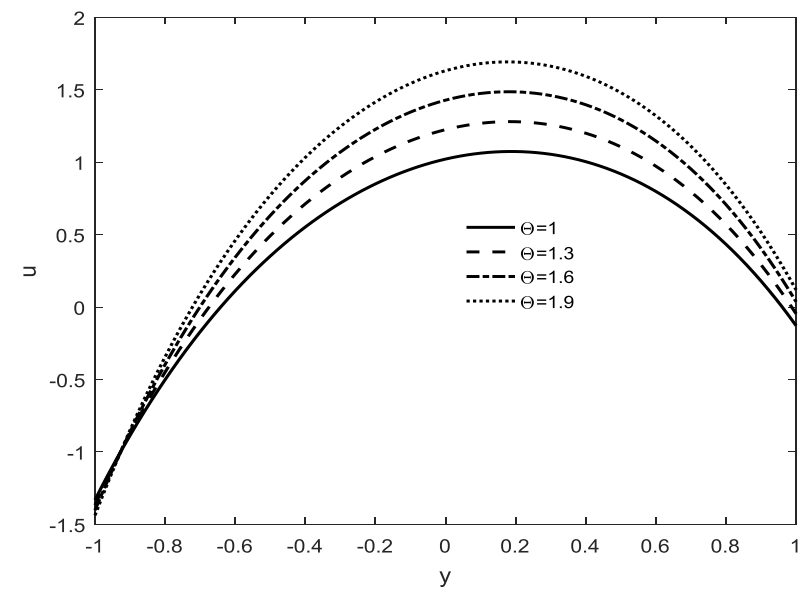

Fig. 21: Influence of mean flow rate on velocity profiles.

\section{Appendix - A}

$$
\begin{aligned}
& p_{1}=\frac{1+N t / N b}{h_{2}-h_{1}+2 L_{2}}, p_{2}=-p_{3} e^{-m_{1} p_{1} h_{1}}\left[1+L_{2} m_{1} p_{1}\right] \text {, } \\
& T^{2}=M^{2}+\sigma^{2} \\
& p_{3}=\frac{1}{\left(e^{-m_{1} p_{1} h_{2}}-e^{-m_{1} p_{1} h_{1}}\right)-L_{2} m_{1} p_{1}\left(e^{-m_{1} p_{1} h_{2}}+e^{-m_{1} p_{1} h_{1}}\right)} \\
& p_{4}=(N t / N b) p_{3} e^{-p_{1} m_{1} h_{1}}-p_{1} h_{1}+L_{3}\left[(N t / N b) m_{1} p_{1} p_{3} e^{-p_{1} m_{1} h_{1}}+p_{1}\right] \text {, } \\
& m_{1}=\frac{\operatorname{Pr} N b}{1+\operatorname{Pr} R n}, m_{2}=\frac{G r m_{1} p_{1} p_{3}-G c \frac{N t}{N b} p_{3} p_{1} m_{1}}{\left(m_{1} p_{1}\right)^{4}-\left(T m_{1} p_{1}\right)^{2}}, \\
& m_{3}=\frac{G c p_{1}}{2 T^{2}}, m_{4}=T \sinh T h_{2}+L_{1} T^{2} \cosh T h_{2} \\
& m_{5}=T \cosh T h_{2}+L_{1} T^{2} \sinh T h_{2}, \\
& m_{6}=e^{-m_{1} p_{1} h_{2}}\left[L_{1} m_{1}^{2} p_{1}^{2} m_{2}-m_{1} p_{1} m_{2}\right] \text {, } \\
& m_{7}=2 m_{3}\left[h_{2}+L_{1}\right], m_{8}=T \sinh T h_{1}+L_{1} T^{2} \cosh T h_{1}, \\
& m_{9}=T \cosh T h_{1}+L_{1} T^{2} \sinh T h_{1} \text {, } \\
& m_{10}=e^{-m_{1} h_{1} p_{1}}\left[L_{1} m_{1}^{2} p_{1}^{2} m_{2}-m_{1} p_{1} m_{2}\right] \text {, } \\
& m_{11}=2 m_{3}\left[h_{1}+L_{1}\right], m_{12}=h_{2}-h_{1} \\
& m_{13}=\cosh T h_{2}-\cosh T h_{1} \text {, } \\
& m_{14}=\sinh T h_{2}-\sinh T h_{1} \text {, } \\
& m_{15}=m_{2}\left(e^{-m_{1} p_{1} h_{2}}-e^{-m_{1} p_{1} h_{1}}\right) \\
& m_{16}=m_{3}\left[h_{2}{ }^{2}-h_{1}{ }^{2}\right], m_{17}=-m_{12}-F \text {, } \\
& m_{18}=m_{4} m_{12}-m_{13}, m_{19}=m_{5} m_{12}-m_{14} \\
& m_{20}=m_{12} m_{6}-m_{15}+m_{7} m_{12}-m_{16}, \\
& m_{21}=m_{8} m_{12}-m_{13}, m_{22}=m_{9} m_{12}-m_{14}
\end{aligned}
$$

$$
\begin{aligned}
& m_{23}=m_{10} m_{12}+m_{11} m_{12}-m_{15}-m_{16} \\
& m_{24}=m_{17}\left(m_{21}-m_{18}\right), m_{25}=m_{19} m_{21}-m_{22} m_{18} \\
& m_{26}=m_{20} m_{21}-m_{23} m_{18}, \\
& p_{5}=\frac{F}{2}-p_{6} h_{2}-p_{7} \cosh T h_{2}-p_{8} \sinh T h_{2}-m_{2} e^{-m_{1} p_{1} h_{2}}-m_{3} h_{2}^{2} \\
& p_{6}=\frac{F-m_{13} p_{7}-m_{14} p_{8}-m_{15}-m_{16}}{m_{12}}, \\
& p_{7}=\frac{m_{17}-m_{19} p_{8}-m_{20}}{m_{18}}, p_{8}=\left(\frac{m_{24}-m_{26}}{m_{25}}\right) .
\end{aligned}
$$

\section{References:}

[1] Latham, T. W. "Fluid motion in a peristaltic pump", MS Thesis, MIT Cambridge (1966). http://hdl.handle.net/1721.1/17282.

[2] Shapiro, A. H., Jaffrin, M. Y., and Weinberg, S. L. "Peristaltic pumping with long wavelengths at low Reynolds number" .Journal of Fluid Mechanics, Vol. 37(4) (1968), pp. 799-825. https://doi.org/10.1017/S0022112069000899

[3] Mishra, M., \& Ramachandra Rao, A. "Peristaltic transport of a Newtonian fluid in an asymmetric channel". ZAMP, Vol. 54 (2003), pp. 532-550. https://doi.org/10.1007/s00033-003-1070-7.

[4] Vajravelu, K., Sreenadh, S., \& Ramesh Babu, V. "Peristaltic transport of a Herschel-Bulkley fluid in an inclined tube". International Journal of Non-Linear Mechanics, Vol. 40(1) (2005), pp. 8390. https://doi.org/10.1016/j.ijnonlinmec.2004.07.001

[5] Vajravelu, K., Sreenadh, S., and Ramesh Babu, V. "Peristaltic pumping of Herschel - Bulkley fluid in a channel”. Appl. Math. $\begin{array}{lllll}\text { Comp., } & \text { Vol. } & 169 & \text { (2005), } & \text { pp. }\end{array}$ http://doi.10.1016/j.amc.2004.09.063.

[6] Hayat, T., Qureshi, M. U., and Hussain, Q. ” Effect of heat transfer on the peristaltic flow of an electrically conducting fluid in a porous space" .Applied Mathematical Modeling, Vol. 33(4) (2009), pp. 1862-1873.

[7] Srinivas, S., \& Muthuraj, R.” Effects of chemical reaction and space porosity on MHD mixed convective flow in a vertical asymmetric channel with peristalsis" .Mathematical and Computer Modeling, Vol. 54(5-6) (2011), pp. 1213-1227. https://doi.org/10.1016/j.mcm.2011.03.032.

[8] Takagi, D.,and Balmforth, N.J. " Peristaltic pumping of rigid objects in an elastic tube". Journal of Fluid Mechanics, Vol. 672 (2011), pp. 219-244. https://doi.org/10.1017/S0022112010005926

[9] Tripathi, D., Anwar Beg, O., A" study on peristaltic flow of nanofluids: Application in drug delivery systems". International Journal of Heat and Mass Transfer, Vol. 70 (2014), pp. 61-70 https://doi.org/10.1016/j.ijheatmasstransfer.2013.10.044.

[10] Vajravelu, K., Sreenadh, S., Lakshminarayana, P., Sucharitha, G., \& Rashidi, M.M. "Peristaltic flow of Phan-Thien-Tanner fluid in an asymmetric channel with porous medium". Journal of Applied Fluid Mechanics, Vol. 9(4) (2016), pp. 1615 - 1625 http://doi.10.18869/acadpub.jafm.68.235.24709

[11] Sreenadh, S., Srinivas, A.N.S., and Selvi, C.K. "Analytical solution for peristaltic flow of conducting nanofluid in an asymmetric channel with slip effect of velocity, temperature and concentration". Alexandria Engineering Journal, Vol. 55(2) (2016), pp. 1085-1098. https://doi.org/10.1016/j.aej.2016.03.015

[12] Srinivas, A. N. S., Selvi, C. K., \& Sreenadh, S. "Peristaltic pumping of a generalized Newtonian fluid in an elastic tube". Journal of Applied Fluid Mechanics, Vol. 10(6) (2017), pp. 1785-1798. http://doi. 10.18869/acadpub.jafm.73.243.27908.

[13] Choi, S.U.S., and Eastman, J.A.” Enhancing thermal conductivity of fluids with nanoparticles". ASME International Mechanical Engineering Congress \& Exposition, San Francisco, CA, pp: 1217(1995).

[14] Buongiorno, J." Convective transport in nanofluids". Journal of Heat Transfer, Vol. 128(3) (2006), pp.240-250. doi:10.1115/1.2150834.

[15] Sheikholeslami,M., Gorji-Bandpay,M., \& Ganji, D.D.” Magnetic field effects on natural convection around a horizontal circular cylinder inside a square enclosure filled with nanofluid". International 
Communications in Heat and Mass Transfer, Vol. 39(7) (2012), pp. 978-986. https://doi.org/10.1016/j.icheatmasstransfer.2012.05.020.

[16] Noreen, S. "Mixed convection peristaltic flow of third order nanofluid with an induced magnetic field". PLoS ONE, Vol. 8(12) (2013), https://doi.org/10.1371/journal.pone.0078770.

[17] Ebaid, A., \& Emad H. Aly, "Exact analytical solution of the peristaltic nanofluids flow in an asymmetric channel with flexible walls and slip condition: Application to the cancer treatment". Computational and Mathematical Methods in Medicine, Article Id: 825376 (2013), pp. 1-8. http://dx.doi.org/10.1155/2013/825376

[18] Nadeem, S., Shaheen, A., \& Hussain, S. "Physiological breakdown of Jeffrey six constant nanofluid flow in an endoscope with nonuniform wall". Aip Advances, Vol. 5 (2015), pp. 127143,1-16. https://doi.org/10.1063/1.4939592.

[19] Kothandapani, M., \& Prakash, J. "The peristaltic transport of carreau nanofluid under effect of a magnetic field in a tapered asymmetric channel: Application of the cancer therapy". Journal of Mechanics and Medicine and Biology, Vol. 15(3) (2015), pp. 1550030-1550032. https://doi.org/10.1142/S021951941550030X.

[20] Dhanapal, C., Kamalakkannan, J., Prakash, J., \& Kothandapani, M. "Analysis of peristaltic motion of a nanofluid with wall shear stress, microrotation, and thermal radiation effects".Applied Bionics and Biomechanics, Article ID 4123741 (2016), pp. 115.http://dx.doi.org/10.1155/2016/4123741

[21] Prakash, D., \& Suriyakumar, P. "Transient hydromagnetic convective flow of nanofluid between asymmetric vertical plates with heat generation". International Journal of Pure and Applied Mathematics, Vol. 113(12) (2017), pp. 1- 10. http://www.ijpam.eu

[22] Nabil T. M. Eldabe, Osama M. Abo-Seida, Adel A. S. Abo-Seliem, ElShekhipy, A.A., \& Nada Hegazy, "Peristaltic Transport of Magnetohydrodynamic Carreau nanofluid with Heat and Mass Transfer inside Asymmetric Channel" .American Journal of Computational $\begin{array}{lllll}\text { Mathematics, Vol. 7(1) (2017), pp. 1-20 } & \end{array}$ DOI: 10.4236/ajcm.2017.71001.

[23] Misra, J.C., Mallick, B., \& Sinha, A. "Heat and mass transfer in asymmetric channels during peristaltic transport of an MHD fluid having temperature-dependent properties". Alexandria Engineering Journal, Vol. 57(1) (2018), pp. 391-406. https://doi.org/10.1016/j.aej.2016.09.021. 